\title{
Drogas na adolescência, comprometimento biopsicossocial e o papel do médico em escolas: relato de experiência
}

\author{
Drugs in adolescent, commitment bio-psychosocial and \\ physician's role in schools: experience report \\ Medicamentos en la adolescencia, compromiso biopsicosocial y \\ el papel del médico en las escuelas: reporte de experiencia
Lara Miranda Rodrigues da CUNHA ${ }^{1}$
Bruna Dias MENEZES ${ }^{\mathbf{1}}$
Allana Baleeiro DANTAS 1
Samara Menali Pereira CAPRONI ${ }^{\mathbf{1}}$
Ciderleia Castro de LIMA2 \\ ${ }^{1}$ Graduanda, Curso de Medicina da Universidade José do Rosário Vellano - UNIFENAS, 37132-440, Alfenas-MG, Brasil \\ ${ }^{2}$ Professora Msc. Curso de Medicina da Universidade José do Rosário Vellano-UNIFENAS, \\ 37132-440, Alfenas-MG, Brasil
}

\begin{abstract}
Resumo
Introdução: O estudo versa sobre a experiência de acadêmicos de medicina ao ministrarem palestras na integração de conhecimentos adquiridos e sua contribuição social, cujo tema central de abordagem foi "Drogas na Adolescência". Objetivo: relatar a experiência de estudantes de medicina do terceiro período na condução de palestras para adolescentes em escolas de ensino fundamental e médio, com a abordagem temática "Drogas na adolescência, comprometimento biopsicossocial e o papel do médico em escolas". Material e Método: Relato de experiência, elaborado com base nas experiências compartilhadas em palestras ministradas aos adolescentes de ensino fundamental e médio do sul de Minas Gerais. Resultado: A aproximação do universo adolescente possibilita conhecer o quanto eles são suscetíveis ao meio, e como eles enfrentam suas inquietações sobre a temática. Poucos são os que participam integralmente, outra parcela sente-se desafiado ou mesmo violados quando sujeitos a receberem informações que a princípio julga saber tudo. Conclusão: As escolas possuem um papel fundamental na formação dos jovens, acredita-se que se possibilitarem o trabalho conjunto de outros profissionais de áreas afins para auxiliar e discutir os aspectos da drogadição, bem como, seus malefícios poderá ser o caminho para a redução do uso das drogas (lícito-ilícitas), com a orientação informa-se, conscientiza-se e garante a saúde integral dos adolescentes, sem prejuízos na fase adulta e para a sociedade.
\end{abstract}

Descritores: Adolescente; Educação; Medicina Preventiva; Transtornos Relacionados ao Uso de Substâncias.

\begin{abstract}
Introduction: The study deals with the experience of medical academics when they lecture on the integration of acquired knowledge and their social contribution, whose main theme was "Drugs in Adolescence". Objective: To report the experience of medical students of the third period in conducting lectures for adolescents in primary and secondary schools, with the thematic approach "Drugs in adolescence, biopsychosocial commitment and the role of the doctor in schools". Material and Method: Report of experience, elaborated based on the experiences shared in lectures given to the adolescents of primary and secondary education in the south of Minas Gerais. Result: The approach of the adolescent universe makes it possible to know how susceptible they are to the environment, and how they face their concerns about the subject. Few are those who participate fully, another portion feels challenged or even violated when subjected to receiving information that at first judges to know everything. Conclusion: Schools have a fundamental role in the training of young people. It is believed that it will be possible to work together with other professionals in related fields to help and discuss aspects of drug addiction, as well as their Use of drugs (licit-illicit), with the guidance informs, is aware of and guarantees the integral health of adolescents, without harm in adulthood and society.

Descriptors: Adolescent; Education; Preventive Medicine; Substance-Related Disorders.
\end{abstract}

\section{Resumen}

Introducción: El estudio se refiere a la experiencia médica estudiantes dando conferencias a la integración de los conocimientos adquiridos y su contribución social, cuyo enfoque tema central fue "Las drogas en adolescentes." Objetivo: Presentar la experiencia de los estudiantes de medicina tercer período en la realización de conferencias para adolescentes en las escuelas primarias y secundarias, con las temáticas de aproximación "drogas en la adolescencia, el compromiso biopsicosocial y el papel del médico en las escuelas." Material y Métodos: Experiencia informe, preparado sobre la base de las experiencias compartidas en las clases a los adolescentes de la secundaria y en el sur de Minas Gerais. Resultado: El enfoque del universo de los adolescentes hace posible saber lo mucho que son susceptibles al medio ambiente, y cómo hacer frente a sus preocupaciones sobre el tema. Pocos son los que participan plenamente, otra parte se deja interpelar o violados cuando se someten a la recepción de la información que el principio entiende todo. Conclusión: Las escuelas tienen un papel clave en la formación de los jóvenes, se cree que si permiten el trabajo conjunto de otros profesionales en campos relacionados para asistir y discutir los aspectos de la adicción a las drogas, así como sus malas acciones pueden ser la manera de reducir uso de drogas (lícitas-ilícitas), con la orientación que se informa, se da cuenta y asegura que el estado general de salud de los adolescentes sin pérdida en la edad adulta y la sociedad.

Descriptores: Adolescente; Educación; Medicina Preventiva, Trastornos Relacionados con Sustancias.

\section{INTRODUÇÃO}

As pesquisas epidemiológicas mostram que o uso e abuso de drogas aumentam em ritmo acelerado, sendo na adolescência que, em geral, inicia-se o consumo ${ }^{1,2}$.

Isso deve ao fato de ser nesse período da vida que a maioria dos jovens não acata orientações e ainda acredita que possa tomar as próprias decisões e administrar a própria vida. Nesta etapa o indivíduo se distancia do âmbito familiar.

Abadi $^{3}$ destaca que o uso de drogas pode ser visto como uma forma pela qual o adolescente procura se rebelar, 
no desafio contra a autoridade representada pela família, pelas leis e pela sociedade ${ }^{3}$.

Outros estudos discutem o fato de os adolescentes que querem iniciar ou aumentar o uso de drogas procuram por grupos com valores e comportamentos semelhantes. Desse modo, pesquisas corroboram que a influência de amigos e colegas, não pode ser vista na totalidade como o ingresso às drogas, uma vez que o processo envolve fatores individuais, familiares e sociais adversos que se somam aumentando a possibilidade do uso excessivo 4 .

A disponibilidade e a existência de drogas na sociedade em que se vive têm favorecido seu uso pelos adolescentes e jovens, e isto pode ter sido contribuído pela facilidade de acesso às drogas lícitas, nesse caso o cigarro e o álcool na sociedade.

Esse estudo tem como objetivo relatar a experiência de estudantes de medicina do terceiro período na condução de palestras para adolescentes em escolas de ensino fundamental e médio, com a abordagem temática "Drogas na adolescência, comprometimento bio-psicossocial e o papel do médico em escolas". Versa, portanto, sobre a importância da conscientização no mundo jovem sobre as drogas devido à alta influência sobre eles.

\section{MATERIAL E MÉTODO}

Trata-se de um estudo de abordagem qualitativa, descritivo, realizado por graduandos do terceiro período do Curso de Medicina da Universidade José do Rosário Vellano, Campus de Alfenas, localizada no Sul de Minas Gerais. Realizado com base nas apresentações, em formato de palestra, ministradas para adolescentes do Colégio Prof. Roque N. Tamburini (Colégio Anglo), Colégio CRA, Colégio Atenas, Colégio Sagrado Coração de Jesus, Escola Estaudal Prefeito Ismael Brasil Correa, Escola Estadual Judith Vianna, Escola Estadual Doutor Emílio Silveira, Escola Estadual Napoleão Salles, Escola Estadual Samuel Engel e Escola Estadual Padre José Grimminck, situados na cidade de Alfenas-MG, entre as turmas do $9^{\circ}$ ano do ensino fundamental ( $8^{\mathrm{a}}$ série) e do $1^{\circ}, 2^{\circ}$ e $3^{\circ}$ anos do ensino médio, envolvendo a participação de adolescentes com idades entre 13 e 18 anos.

A apresentação de palestras foi escolhida como a melhor forma encontrada para levar o assunto ao públicoalvo devido à facilidade na abordagem do tema e a possibilidade de estimular a discussão entre os alunos, solucionando as dúvidas e problemáticas apresentadas por eles.

Para fomentar as discussões buscou-se apoio nos acervos bibliográficos, tais como os livros, os jornais e, sobretudo, na análise de artigos científicos publicados nas bases de dados disponibilizados pela Biblioteca Virtual de Saúde - BVS

\section{RESULTADOS}

A exposição foi organizada em várias palestras, realizadas ao longo do primeiro semestre do ano de 2015 nas escolas de ensino fundamental e médio. $\mathrm{O}$ desenvolvimento do trabalho foi norteado pela atuação participativa dos adolescentes, que se mostraram questionadores em sua minoria. As dúvidas foram esclarecidas conforme as perguntas surgiam no decorrer das palestras. Para a motivação participativa, utilizou de recursos didáticos como o Datashow, os vídeos e os textos contendo depoimentos de usuários e ex-usuários.
Ao início da explanação sobre assuntos relacionados às drogas no geral, adotou-se uma pergunta descritiva e ampla: "Na opinião de vocês, qual o significado da palavra droga?". Colocado isso em evidência, foram atribuídos vários significados às drogas com base em suas vivências. Cada participante do grupo foi então convocado a falar brevemente sobre subtemas pré-determinados como composição, aspectos biológicos, quadro clínico, tolerância, intoxicação, síndrome de abstinência, tratamento e reabilitação de usuários de drogas. Ao término, foi solicitado aos alunos que expusessem suas dúvidas, deixando em aberto o espaço para procurar algum integrante do grupo para a discussão.

O tempo médio de cada palestra foi de 20 minutos, além dos momentos abertos para discussão em cada turma. Ao término da apresentação, foi distribuído para os alunos um panfleto informativo com depoimentos de ex-usuários, que contavam como eles haviam conhecido o mundo das drogas e como conseguiram se livrar dele.

As palestras resultaram em outras inquietações para os estudantes de medicina, enquanto formadores de opiniões, tais como estratégias para reabilitação dos indivíduos na sociedade e sua real eficácia.

O trabalho permitiu ao grupo conhecer as dúvidas e anseios dos alunos com relação às drogas e possibilitou a orientação e o direcionamento de forma compreensível aos mesmos. Também criou um momento de troca de experiências e um espaço para a exposição de opiniões.

\section{DISCUSSÃO}

A adolescência, fase intermediária entre a infância e a juventude, conhecida como o período das grandes mudanças intrínsecas, caracteriza-se por mudanças biopsicossociais e exteriorização de conflitos em virtude da maior labilidade emocional e da sensibilidade aumentada, o que confere à pessoa certo desconforto. Com isso, surgem dúvidas e questões de várias ordens, desde como viver a vida, modo de ser, de estar com os outros, até a construção do futuro relacionado às escolhas. Essas características e situações fazem com que ele fique vulnerável a inúmeros riscos, dentre os quais se podem citar o uso de drogas tanto lícitas quanto ilícitas 5 .

Os adolescentes que experimentam drogas dão motivos similares às descritas para o fumo e para o álcool como a pressão dos companheiros, o uso por alguns dos familiares, o estresse, o descontentamento, a rebeldia característica da fase, a ansiedade, a depressão e a baixa autoestima. O uso do fumo e do álcool em geral, representa um marco para a experimentação de outras drogas ${ }^{6}$. E, por isso, com a falta de conscientização e informação desses jovens sobre as consequências do uso dessas substâncias, eles ficam susceptíveis a elas, já que ainda estão em fase de formação e não possuem maturidade o suficiente para conseguir enxergar com os próprios olhos o abismo que há nessa escolha, bem como quando se desiste encarar as dificuldades da realidade.

Outrossim, o estudo mostrou que, apesar do bombardeio de informações a respeito do perigo do fumo, do álcool e das drogas, nenhum adolescente fica imune à influência social e ao fácil acesso. Isto é em potencial mais elevado nos casos em que os pais fumam e/ou bebem em excesso ou faz uso de outros tipos de drogas ${ }^{6}$.

Devido a sua condição de pessoa em desenvolvimento, o jovem traz em si uma condição 
intrínseca de vulnerabilidade, precisando assim, de proteção física, psíquica e moral, com total atenção ${ }^{7}$.

Neste contexto, sabe-se que existe predisposição quando ocorre o desinteresse da pessoa em relação a casos de perigo; a falta de acesso aos serviços e/ou informações e ausência de autoconfiança para sustentar ou implementar mudanças no próprio comportamento. A vulnerabilidade pode ser entendida como o produto da interação entre suas características cognitiva, afetiva, psíquica e estruturas sociais de desigualdade de gênero, classe social e raça, determinando acessos, oportunidades e produzindo sentidos para o sujeito sobre ele mesmo e o mundo ${ }^{7}$.

Para tanto, na abordagem e teorias da educação no aspecto cognitivista, defendido por Jean Piaget [1920], o indivíduo está sujeito a um contínuo aprendizado, na interação com o meio em que se encontra inserido. Com relação às drogas, a sua inserção pode acontecer de forma gradativa, contudo, evoluir e repercutir casos com quadros de dependência.

Vale ressaltar que a dependência química é caracterizada pelo consumo de droga desregrado, onde o usuário não consegue se abdicar da droga. Concomitantemente a dependência, se inicia o processo de tolerância, em que a pessoa necessita utilizar cada vez doses maiores da substância para sentir o mesmo efeito gratificante que antes sentia com doses menores. Ou seja, nesse momento, a substância psicoativa já iniciou seu quadro de feedback positivo e, quanto mais o individuo a consome, mais ele vai querer consumir. Assim, a compulsão e a fissura são frequentes e cada vez mais intensas.

Estudo demonstra que todas as drogas levam a algum tipo de tolerância em maior ou menor grau. O processo de se transpor de um nível para o outro de tolerância se torna constante e leva ao aumento de substâncias consumidas e de drogas leves passam a usar as drogas mais pesadas ${ }^{8}$.

Ainda, o autor ressalta que a síndrome de abstinência pode ser iniciada quando o usuário passa por um determinado período de tempo sem ingerir a substância, tendo assim, seu corpo reagindo pela ausência da droga no organismo.

O problema é que com o quadro de tolerânciadependência instalado, a pessoa não só agrava o seu estado de saúde, mas também começa a criar mais problemas no seu meio, na tentativa de consumir a substância que o deixa em estado pleno de prazer. E, com isso apresenta comportamentos agressivos no âmbito familiar, junto aos amigos e/ou a qualquer pessoa de seu convívio e, para a segurança social, com os desvios de comportamento instalados, pode significar um risco social.

Nessa perspectiva, faz saber que todo usuário pode vir a tornar um pequeno traficante com vista a sustentar seu vício.

Estudo corrobora que, os usuários são levados a roubar, assaltar ou matar, caso não consigam saldar suas dívidas com aqueles que os ameaçam em tirar suas vidas. Já outros, podem participar das quadrilhas, seja para pagarem suas dívidas, seja para se sentirem imbatíveis por aqueles que os ameaçam 9 .

Diante disso, estudo tem mostrado que a quantidade de adolescentes que morrem no exercício de suas atividades no tráfico de drogas, desejando ascensão no plano de carreira, ou seja, com o ideal de ocupar um lugar mais destacado na estrutura do tráfico é impactante ${ }^{9}$.

Então, deve-se reforçar o papel que a escola exerce na formação desses jovens no intuito de reduzir a incidência dos mesmos à criminalidade. Com isso, a escola ocupa um espaço importante no desenvolvimento de atividades de orientação, de liberdade de expressão e de educação em saúde. Para tal, a educação em saúde, nesse espaço, deve contar, primeiramente, com a participação dos profissionais nela inseridos, os quais podem contribuir nas abordagens inovadoras de educação, promovendo a compreensão do fenômeno saúde $\mathrm{e}^{10,11}$.

Assim, inserir temas relacionados à saúde nas escolas é parte das políticas públicas para o fortalecimento da ação comunitária, priorizando a participação das pessoas no contexto escolar, visando desenvolver habilidades em lidar com diferentes informações, como as drogas ${ }^{12}$.

Diante desse contexto, os adolescentes, alvo fácil da sociedade, requer abordagens que proporciona a desconstrução de significados em relação às drogas e o restabelecimento da saúde na sua integralidade. Para tal, o profissional de saúde deve conhecer o tratamento e compreendê-lo por meio dos significados atribuídos a ele pelos usuários sem efetuar pré-conceitos envolvendo o meio familiar, escolar e social ${ }^{13}$.

Nessa perspectiva, em atividade com os alunos, pode observar que o corpo administrativo dos colégios apresentou-se aberto para novas abordagens que contribuam para melhor informar e formar os alunos. Contudo, o corpo docente em sua maioria mostrou-se desinteressado, não participando integralmente das oficinas. Quanto aos adolescentes, talvez por tratar de um tema exposto frequentemente pela mídia e não ter uma representação social solidificada pelos adolescentes, em sua maioria, mesmo presentes nas oficinas, os adolescentes não demonstraram adesão às orientações.

Por meio do presente estudo, baseado na experiência do grupo após as discussões com os alunos e na percepção da importância do tema, observou-se a importância da abordagem de forma direta e clara com adolescentes, visando sempre promover a educação e a saúde.

Percebeu-se, portanto, a necessidade da realização de novos trabalhos de discussão e conscientização dos adolescentes pelos profissionais da área de saúde, especialmente o médico, através de intervenções na comunidade e nas escolas.

Diante disso, aos futuros médicos reservam-se desafios com potencialidades de problemas de desvios de comportamento.

\section{CONCLUSÃO}

Com as palestras foi possível verificar pouco interesse na apresentação, talvez por ter sido compreendido como um caráter de orientação, desafiando com isso a fase que confere ao adolescente uma pseudoautonomia. Os profissionais da educação também mostraram desinteresse, atribuído ao fato de não tomarem para si a parcela de responsabilidade social.

Abordar sobre drogas muitas vezes é visto pelos adolescentes como algo comum, natural e banal, principalmente no que diz respeito às drogas lícitas em que o acesso é fácil, e geralmente não há preocupação com as consequências sobre a saúde do indivíduo.

Apesar da maioria dos alunos não se interessarem pela palestra, acreditamos que aqueles que participaram integralmente das palestras entenderam as principais consequências que as drogas podem trazer para o organismo.

Mesmo a contragosto, as drogas fazem parte do cotidiano e não discriminam raça e/ou classe social. Explicamos sobre os seus efeitos a curto, médio e longo prazo, demonstrando quais são os fatores que influenciam os adolescentes a iniciarem o uso das drogas, como problemas 
no relacionamento familiar; fácil acesso e preço acessível; e como a legislação vislumbra os usuários de drogas.

E como já reforçado em eventos científicos, a apresentação desse tema associado a imagens visuais contribui para a persuasão e melhor adesão às orientações feitas em grupo e oficinas para adolescentes.

Ao que se sabe, no Brasil não é prática frequente profissionais de saúde palestrarem sobre drogas em colégios de ensino médio e fundamental. Geralmente o tema é atribuído ao setor de segurança pública, daí profissionais como os policiais abordarem a temática.

Visto isto, é preciso priorizar políticas preventivas, gerando projetos mais ajustados e articulados entre os diversos setores para a realidade brasileira. Os indivíduos que já se encontram no mundo das drogas precisam de um tratamento psicológico e medicamentoso juntamente com o apoio familiar e do ambiente escolar, cabendo a este papel fundamental no desenvolvimento de trabalhos preventivos para a formação dos jovens.

\section{REFERÊNCIAS}

1. Carlini EA, Carlini-Cotrim BHRS, Silva-Filho AR. Sugestões para programas de prevenção ao abuso de drogas no Brasil. São Paulo: CEBRID: São Paulo; 1990.

2. Carlini EA, Cotrim BC. Perfil epidemiológico do usuário de substâncias psicoativas em escolas estaduais de Porto Alegre/1992. Porto Alegre: Relatório não publicado; 1994.

3. Abadi S. Adolescence et drogue, un symptôme dans la civilisation. Psychanalyse dans la Civilization. 1991; 4:603-13.

4. Fergusson DM, Horwood LJ. Prospective childhood predictors of deviant peer affiliations in adolescence. $\mathrm{J}$ Child Psychol Psychiatry. 1999; 40(4):581-92.

5. Zeitoune RCG, Ferreira VS, Silveira HS, Domingos AM, Maia AC. O conhecimento de adolescentes sobre drogas lícitas e ilícitas: uma contribuição para a enfermagem comunitária. Esc Anna Nery. 2012; 16(1):57-63.

6. Silber TJ, Souza RP. Uso e abuso de drogas na adolescência: o que se deve saber e o que se pode fazer. Adolesc. Latinoam. 1998;1(3):148-62.

7. Pessalacia JDR, Menezes ES, Massuia D. A vulnerabilidade do adolescente numa perspectiva das políticas de saúde pública. Bioethikos. 2010; 4(4):423-30.

8. Santos TC, Carrapato JL. As consequências do uso de substancias psicoativas no aspecto biopsicossocial. Araçatuba (SP): Centro Universitário Toledo; 2010. Disponível em: http://intertemas.unitoledo.br/ revista/index.php/ETIC/article/viewFile/2196/2355

9. Pereira SEFN. Redes sociais de adolescentes em contexto de vulnerabilidade social e sua relação com os riscos de envolvimento com o tráfico de drogas [tese]. Brasília: Curso de Psicologia/Universidade Nacional de Brasília; 2009.

10. Oliveira DL. A "nova" saúde pública e a promoção da saúde via educação: entre a tradição e a inovação. Rev Latino-Am Enfermagem. 2005;13(3):605-13.

11. Besen CB, Souza Netto M, Da Ros MA, Silva FW, Silva CG, Pires MF. A estratégia saúde da família como objeto de educação em saúde. Saude Soc. 2007; 16(1):57-68.

12. Aerts D, Alves GG, La Salvia MW, Abegg C. Promoção de saúde: a convergência entre as propostas da vigilância da saúde e da escola cidadã. Cad. Saúde Pública. 2004 20(4):1020-8.

13. Paula ML, Jorge MSB, Albuquerque RA, Queiroz LM. Usuário de crack em situações de tratamento: experiências, significados e sentidos. Saude soc. 2014; 23(1): 118-30.

\section{CONFLITO DE INTERESSES}

Os autores declaram não haver conflitos de interesse.

\section{AUTOR PARA CORRESPONDÊNCIA}

Lara Miranda Rodrigues da Cunha

laramiranda@hotmail.com

Submetido em 14/03/2017

Aceito em 02/05/2017 